\title{
Multiple Shift SBR2 Algorithm for Calculating the SVD of Broadband Optical MIMO Systems
}

\author{
Zeliang Wang*, André Sandmann ${ }^{\dagger}$, John G. McWhirter* and Andreas Ahrens ${ }^{\dagger}$ \\ ${ }^{*}$ School of Engineering, Cardiff University, Cardiff, Wales, UK \\ ${ }^{\dagger}$ Hochschule Wismar, University of Applied Sciences-Technology, Business and Design, Wismar, Germany \\ \{WangZ49, McWhirterJG\}@ $@$ cardiff.ac.uk, A.Sandmann@stud.hs-wismar.de, Andreas.Ahrens@hs-wismar.de
}

\begin{abstract}
Polynomial matrix singular value decomposition (PSVD) plays a very important role in broadband multiple-input multiple-output (MIMO) systems. It can be used to decompose a broadband MIMO channel matrix in order to recover the transmitted signals corrupted by the channel interference (CI) at the receiver. In this paper, a novel algorithm, known as multiple shift second order sequential best rotation (MS-SBR2), is proposed to compute the approximate PSVD. Simulations are implemented under a $(2 \times 2)$ optical MIMO channel model. Bit error rate (BER) performances are evaluated among different transmission schemes. In addition, power allocation (PA) scheme is investigated to further optimize the BER performance.

Keywords-Broadband MIMO, Multiple Shift SBR2, PSVD.
\end{abstract}

\section{INTRODUCTION}

An explosive development of MIMO technology has been witnessed in wireless communication systems over the last decade. Compared to single-input single-output (SISO) systems, MIMO systems are capable of achieving higher data rates and transmission reliabilities. Aiming to increase the fiber capacity, the concept of MIMO in optical transmission systems has also attracted intensive research interests [1], [2].

In broadband MIMO systems, the channel is characterized by frequency-selective fading. In order to recover the transmitted data sequence corrupted by channel interference (CI), a conventional method is to combine the spatio-temporal vector coding (STVC) [3], [4] with the singular value decomposition (SVD) based equalization technique [5] . However, there are some existing papers [6], [7] which discussed an alternative signal pre- and post-processing method used in broadband MIMO systems. Basically this method consists of two steps. The first step is based on the PSVD which is used to remove the CI by decomposing the frequency-selective MIMO channel into a number of independent frequency-selective SISO channels, and the second step involves removing the remaining inter-symbol interference (ISI), which can be implemented by further equalization techniques, such as zero-forcing (ZF) equalization or maximum likelihood sequence estimations (MLSE).

There are different ways of calculating the PSVD of a polynomial matrix, such as using polynomial matrix QR

The authors would like to thank the Engineering and Physical Sciences Research Council (EPSRC) Grant number EP/K014307/1 and UK MOD University Defence Research Collaboration in Signal Processing for partially supporting this work. decomposition to formulate the PSVD [8], PSVD based on generalized Kogbetliantz transformations [9], and PSVD by polynomial matrix eigenvalue decomposition (PEVD) method [10], which is analogous to how the scalar matrix eigenvalue decomposition (EVD) can be used to generate the singular value decomposition (SVD) of a matrix. In terms of the PSVD by PEVD method, the second order sequential best rotation (SBR2) algorithm [11] has been used in the existing literature. However, an improved version of the SBR2 algorithm, i.e. MS-SBR2 [12], has been recently proposed by the authors for calculating the PEVD of polynomial matrices. The improved algorithm can provide much faster convergence than the SBR2 algorithm when dealing with high dimension polynomial matrices. In other words, the diagonalization of bigger MIMO channel matrices can be implemented faster than that of using the SBR2 algorithm.

The novelty of this work is to exploit the proposed MSSBR2 algorithm in the application of solving the channel equalization problem for broadband MIMO systems. Our simulations are implemented based on a measured $(2 \times 2)$ optical MIMO channel which comprises a $1.4 \mathrm{~km}$ multi-mode fiber and optical couplers at both ends, and the channel impulse responses are measured for the operating wavelength of $1576 \mathrm{~nm}$ [7]. In particular, transmission and power allocation schemes are employed to bring further improvement with respect to the BER performance.

The rest of the paper is structured as follows. The optical MIMO channel model with polynomial matrix representation is described in Sec. II. In Sec. III we introduce the idea of broadband MIMO channel decomposition, i.e. PSVD. Sec. IV presents the proposed MS-SBR2 algorithm for calculating the PSVD. Simulation results and conclusions are shown in Sec. V and Sec. VI, respectively.

\section{MIMO ChANNEL MODEL}

Given a frequency selective optical MIMO link with $n_{\mathrm{T}}$ optical inputs and $n_{\mathrm{R}}$ optical outputs, the channel can be modeled as a polynomial matrix with an indeterminate variable $z^{-1}$ given by

$$
\underline{\mathbf{C}}(z)=\sum_{\tau=0}^{T} \mathbf{C}[\tau] z^{-\tau}=\left[\begin{array}{ccc}
\underline{c}_{11}(z) & \cdots & \underline{c}_{1 n_{\mathrm{T}}}(z) \\
\vdots & \ddots & \vdots \\
\underline{c}_{n_{\mathrm{R}} 1}(z) & \cdots & \underline{c}_{n_{\mathrm{R}} n_{\mathrm{T}}}(z)
\end{array}\right],
$$


where $\tau, T \in \mathbb{Z}$ and $\mathbf{C}[\tau] \in \mathbb{C}^{n_{\mathrm{R}} \times n_{\mathrm{T}}}$ denotes the polynomial coefficient matrix at time lag $\tau$ and $\underline{c}_{\nu \mu}(z)$ is the polynomial matrix entity which represents the channel impulse response between the $\mu$-th optical input and the $\nu$-th optical output. It takes the form of

$$
\underline{c}_{\nu \mu}(z)=\sum_{\tau=0}^{T} c_{\nu \mu}[\tau] z^{-\tau},
$$

where $c_{\nu \mu}[\tau]$ denotes a non-zero element of the symbol rate sampled overall channel impulse response at the $\tau$-th lag. In this case there are $T+1$ lags in total for each SISO channel. Throughout this paper, polynomial matrices and vectors are denoted as underscored boldface letters. Finally, the resulting MIMO system model can be described in polynomial matrix notation as follows

$$
\underline{\mathbf{x}}(z)=\underline{\mathbf{C}}(z) \underline{\mathbf{s}}(z)+\underline{\mathbf{n}}(z),
$$

where $\underline{\mathbf{x}}(z), \underline{\mathbf{s}}(z)$ and $\underline{\mathbf{n}}(z)$ represent the received signal, the source signal and the noise signal in $z$-domain respectively.

\section{BRoAdBAND MIMO CHANNEL DECOMPOSITION VIA PSVD}

One potential application of PSVD is to enable communication over a broadband MIMO system in which the channel matrix is represented by a polynomial matrix as shown in equation (1). In this case, provided the channel matrix has firstly been estimated, the PSVD then can be used to simplify a MIMO channel equalization problem into a set of SISO problems. In other words, the CI can be removed by performing the PSVD to the channel matrix $\underline{\mathbf{C}}(z)$, which can be expressed as [10]

$$
\underline{\mathbf{C}}(z)=\underline{\widetilde{\mathbf{U}}}(z) \underline{\boldsymbol{\Sigma}}(z) \underline{\mathbf{V}}(z)=\underline{\widetilde{\mathbf{U}}}(z)\left[\begin{array}{c}
\boldsymbol{\Gamma}(z) \\
0
\end{array}\right] \underline{\mathbf{V}}(z),
$$

where we assume $n_{\mathrm{R}} \geq n_{\mathrm{T}}$, and $\underline{\boldsymbol{\Gamma}}(z)$ is a diagonal polynomial matrix with $n=n_{\mathrm{T}}$ diagonal elements, s.t. $\underline{\boldsymbol{\Gamma}}(z)=\operatorname{diag}\left\{\underline{\gamma}_{1}(z), \underline{\gamma}_{2}(z), \cdots, \underline{\gamma}_{n}(z)\right\}$. $\underline{\mathbf{U}}(z)$ and $\underline{\mathbf{V}}(z)$ are paraunitary polynomial matrices with dimension $n_{\mathrm{R}} \times n_{\mathrm{R}}$ and $n_{\mathrm{T}} \times n_{\mathrm{T}}$ respectively, s.t. $\underline{\widetilde{\mathbf{U}}}(z) \underline{\mathbf{U}}(z)=\underline{\mathbf{U}}(z) \underline{\widetilde{\mathbf{U}}}(z)=\mathbf{I}_{n_{\mathrm{R}}}$ and $\underline{\tilde{\mathbf{V}}}(z) \underline{\mathbf{V}}(z)=\underline{\mathbf{V}}(z) \underline{\tilde{\mathbf{V}}}(z)=\mathbf{I}_{n_{\mathrm{T}}}$. Here the notation $\{\sim\}$ over a polynomial matrix denotes the paraconjugate operation which is computed by performing Hermitian transpose $\{\cdot\}^{\mathrm{H}}$ to all the polynomial coefficient matrices $\mathbf{U}[\tau]$ and time-reversing all entries inside, i.e. $\widetilde{\widetilde{U}}(z)=\underline{\mathbf{U}}^{\mathrm{H}}(1 / z)$.

Note that $\underline{\widetilde{U}}(z)$ and $\underline{\underline{V}}(z)$ are acting as the multichannel allpass filters which can transform the frequency selective MIMO channel into a number of independent frequency selective SISO channels while still preserving the total signal energy [13].

In this paper, the PSVD in equation (4) is implemented by calculating the PEVD of two polynomial matrices $\underline{\mathbf{C}}(z) \underline{\widetilde{\mathbf{C}}}(z)$ and $\underline{\widetilde{\mathbf{C}}}(z) \underline{\mathbf{C}}(z)$, which take the form as

$$
[\underline{\mathbf{C}}(z) \underline{\widetilde{\mathbf{C}}}(z)]_{n_{\mathrm{R}} \times n_{\mathrm{R}}}=\underline{\tilde{\mathbf{U}}}(z) \underline{\boldsymbol{\Sigma}}(z) \underline{\tilde{\boldsymbol{\Sigma}}}(z) \underline{\mathbf{U}}(z),
$$

and

$$
[\underline{\widetilde{\mathbf{C}}}(z) \underline{\mathbf{C}}(z)]_{n_{\mathrm{T}} \times n_{\mathrm{T}}}=\underline{\tilde{\mathbf{V}}}(z) \underline{\tilde{\boldsymbol{\Sigma}}}(z) \underline{\boldsymbol{\Sigma}}(z) \underline{\mathbf{V}}(z) .
$$

Further details about the PEVD algorithm will be discussed in the following section. To eliminate the CI, the transmit data vector $\underline{\mathbf{s}}(z)$ is pre-multiplied by $\underline{\widetilde{\mathbf{V}}}(z)$ at the transmitter, and pre-multiplied by $\underline{\mathbf{U}}(z)$ at the receiver, which results in

$$
\underline{\mathbf{x}}(z)=\underline{\boldsymbol{\Sigma}}(z) \underline{\mathbf{s}}(z)+\underline{\mathbf{w}}(z),
$$

where $\underline{\mathbf{w}}(z)=\underline{\mathbf{U}}(z) \underline{\mathbf{n}}(z)$. Note that neither the transmit power is increased, nor the channel noise is enhanced here.

Unlike the conventional SVD-based method, each diagonal element (also called layer) in $\underline{\boldsymbol{\Sigma}}(z)$ is frequency-selective and hence ISI occurs. In order to remove the ISI, layer-specific T-spaced zero forcing equalizers [7] are adopted in this paper.

\section{PSVD USING THE MS-SBR2 ALgORITHM}

As mentioned above, the PEVD method can be used to formulate the PSVD problem in equation (4), and the idea of PEVD has been generalized as [11]

$$
\underline{\mathbf{H}}(z) \underline{\mathbf{R}}(z) \underline{\widetilde{\mathbf{H}}}(z) \approx \underline{\mathbf{D}}(z),
$$

where $\underline{\mathbf{R}}(z)$ is assumed to be a $M \times M$ input para-Hermitian matrix, such that $\underline{\widetilde{\mathbf{R}}}(z)=\underline{\mathbf{R}}(z), \underline{\mathbf{H}}(z)$ is a paraunitary matrix which aims to diagonalize $\underline{\mathbf{R}}(z)$ by means of paraunitary similarity transformation, and $\underline{\mathbf{D}}(z)$ is (ideally) a diagonal matrix. This is an iterative process which transforms all the off-diagonal elements in $\underline{\mathbf{R}}(z)$ onto the diagonal. Several algorithms exist for calculating the PEVD in equation (8) [11], [14], [15], however, this paper is concerned only with the MSSBR2 algorithm previously presented by the authors in [12].

The MS-SBR2 algorithm is an improved version of the SBR2 algorithm in terms of the algorithm convergence speed. Basically it adopts the advantages of less computational cost from SBR2 and the faster convergence from MSME-SMD [15], which seems to provide a compromise between the SBR2 and the SMD algorithm family. The SBR2 algorithm is firstly introduced before we move forward to the MS-SBR2 algorithm.

At the $i$-th iteration, the SBR2 algorithm [11] starts by locating the maximum off-diagonal element $r_{j k}^{(i)}[\tau]$. To find the maximum off-diagonal element, we define a matrix $\mathbf{S}^{(i)}[\tau]$, which contains only the upper triangular elements in $\mathbf{R}^{(i-1)}[\tau]$ with the remaining elements set to zero. Thus the location of $r_{j k}^{(i)}[\tau],(j<k)$ found at $i$-th iteration satisfies

$$
\left\{j^{(i)}, k^{(i)}, \tau^{(i)}\right\}=\arg \max _{j, k, \tau}\left\|\mathbf{S}^{(i)}[\tau]\right\|_{\infty}
$$

where $j^{(i)}, k^{(i)}$ and $\tau^{(i)}$ are the corresponding row, column and time lag index. An elementary delay matrix $\underline{\mathbf{P}}^{(i)}(z)$ and Jacobi rotation $\mathbf{Q}^{(i)}$ are applied to bring $r_{j k}^{(i)}[\tau]$ and its complex conjugate $r_{k j}^{(i)}[-\tau]$ onto the zero-lag $(\tau=0)$ coefficient matrix $\mathbf{R}^{(i-1)}[0]$, and then rotate its energy onto the diagonal. This results in $\underline{\mathbf{R}}^{(i)}(z)$ given by

$$
\underline{\mathbf{R}}^{(i)}(z)=\mathbf{Q}^{(i)} \underline{\mathbf{P}}^{(i)}(z) \underline{\mathbf{R}}^{(i-1)}(z) \underline{\widetilde{\mathbf{P}}}^{(i)}(z) \mathbf{Q}^{\mathrm{H}(i)} .
$$




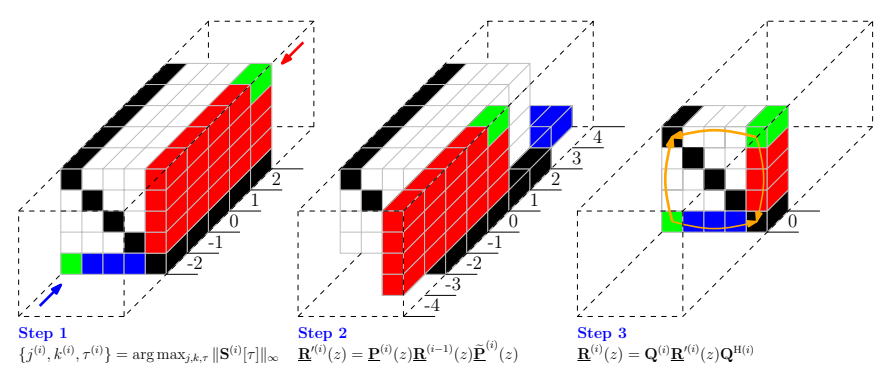

Fig. 1. A 3-dimensional illustration of a $5 \times 5$ polynomial matrix example, showing the $i$-th iteration process using SBR2; Assuming the maximum offdiagonal element $r_{j k}^{(i)}[\tau]$ found is at the location of $\{1,5,2\}$ represented in green color, step 1 shows the location information; Step 2 describes the corresponding row and column shift operations; Step 3 is to transfer the pairwise maximum elements $r_{j k}^{(i)}[\tau]$ and $r_{k j}^{(i)}[-\tau]$ onto diagonal (only zerolag coefficient matrix is shown here for visibility purpose) [11], [15].

A 3-dimensional illustration which shows the procedure of the $i$-th iteration in SBR2 is described in Fig. 1. Thus the elementary paraunitary matrix $\underline{\mathbf{E}}^{(i)}(z)$ can be expressed as

$$
\underline{\mathbf{E}}^{(i)}(z)=\mathbf{Q}^{(i)} \underline{\mathbf{P}}^{(i)}(z)
$$

The algorithm continues its iterative process until all the offdiagonal elements are smaller than a given threshold which can be set to a very small value to achieve sufficient accuracy. Assuming that the algorithm has converged at the $N$-th iteration, the diagonalized para-Hermitian matrix in equation (8) takes the form of

$$
\underline{\mathbf{D}}(z)=\operatorname{diag}\left\{\underline{d}_{1}(z), \underline{d}_{2}(z), \cdots, \underline{d}_{M}(z)\right\},
$$

and the generated paraunitary polynomial matrix is given by

$$
\underline{\mathbf{H}}(z)=\prod_{i=1}^{N} \underline{\mathbf{E}}^{(i)}(z)=\underline{\mathbf{E}}^{(N)}(z) \cdots \underline{\mathbf{E}}^{(2)}(z) \underline{\mathbf{E}}^{(1)}(z) .
$$

However, the MS-SBR2 algorithm uses a distinguishing search strategy of the off-diagonal elements which is akin to that of the MSME-SMD algorithm, so that it can achieve the diagonalization with less iterations than the SBR2 algorithm. For the $i$-th iteration, the MS-SBR2 algorithm involves multiple shifts operations $\underline{\widehat{\mathbf{P}}}^{(i)}(z)$, followed by a sequence of Jacobi rotations $\widehat{\mathbf{Q}}^{(i)}$. Therefore the resulting para-Hermitian matrix is computed by

$$
\underline{\mathbf{R}}^{(i)}(z)=\widehat{\mathbf{Q}}^{(i)} \underline{\widehat{\mathbf{P}}}^{(i)}(z) \underline{\mathbf{R}}^{(i-1)}(z) \underline{\widehat{\mathbf{P}}}^{(i)}(z) \widehat{\mathbf{Q}}^{\mathrm{H}(i)},
$$

where $\underline{\mathbf{\mathbf { P }}}^{(i)}(z)=\prod_{l=1}^{L^{(i)}} \underline{\mathbf{P}}^{(l, i)}(z), \widehat{\mathbf{Q}}^{(i)}=\prod_{l=1}^{L^{(i)}} \mathbf{Q}^{(l, i)}$ and $L^{(i)}$ denotes the total number of off-diagonal elements shifted to the zero-lag coefficient matrix at the $i$-th iteration. Accordingly the elementary paraunitary matrix can be expressed as $\underline{\widehat{\mathbf{E}}}^{(i)}(z)=\widehat{\mathbf{Q}}^{(i)} \underline{\widehat{\mathbf{P}}}^{(i)}(z)$. Note that when $L^{(i)}=1$, the MSSBR2 algorithm is identical to the SBR2 algorithm.

The PEVD algorithms are assessed in terms of the normalized off-diagonal energy $\eta^{(i)}$ at the $i$-th iteration, and it is defined as

$$
\eta^{(i)}=\frac{\sum_{\tau} \sum_{m, n=1, m \neq n}^{M}\left|r_{m n}^{(i)}[\tau]\right|^{2}}{\sum_{\tau}\|\mathbf{R}[\tau]\|_{\mathrm{F}}^{2}}
$$

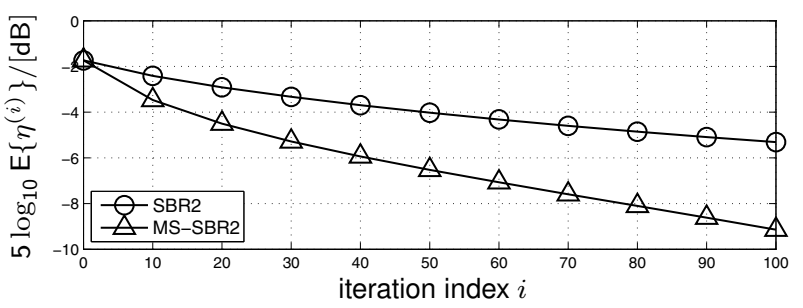

Fig. 2. Comparison of normalized off-diagonal energy $\eta^{(i)}$ between SBR2 and MS-SBR2 algorithms, showing ensemble averages versus iterations.

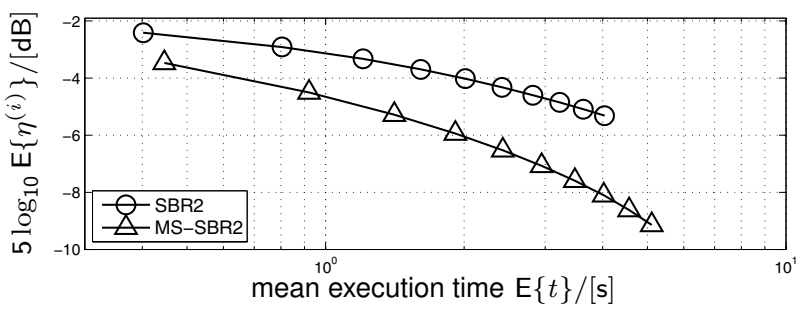

Fig. 3. Comparison of normalized off-diagonal energy $\eta^{(i)}$ between SBR2 and MS-SBR2 algorithms, showing ensemble averages versus mean execution time (measured in MATLAB R2014a on a PC with configurations Intel Core i7-3770T CPU@2.50 GHz and 16 GB RAM).

where the notation $\|\cdot\|_{F}$ denotes the Frobenius norm.

The comparison between these two PEVD algorithms is calculated via Monte Carlo simulations over an ensemble of 100 different random $10 \times 10$ para-Hermitian matrices of order 5, with i.i.d. zero mean unit variance complex Gaussian entries. Fig. 2 shows the normalized off-diagonal energy $\eta^{(i)}$ versus the iteration index $i$. Obviously the MS-SBR2 algorithm requires much fewer iterations than the conventional SBR2 algorithm to achieve the same level of diagonalization. However, it should be noticed that each iteration within MS-SBR2 involves more rotation steps, which means the computational costs between them are comparable.

Nonetheless, the MS-SBR2 algorithm was found to converge faster than SBR2 as shown in Fig. 3. Another motivation of introducing the multiple shift idea into the SBR2 algorithm is that it permits us to minimize the order growth of polynomial matrices by making all row (column) shifts in the same direction, which can potentially reduce the computational cost of the algorithm. This factor is currently being investigated and has not been taken into consideration in this work. For further details of the MS-SBR2 algorithm, see [12].

\section{Simulation Results}

To demonstrate the proposed PSVD method, we applied it to a $(2 \times 2)$ broadband optical MIMO system in which the BER qualities are evaluated over a range of signal-tonoise ratios (SNRs). In addition, different transmission modes for each layer (each SISO channel) are analyzed under a fixed spectral efficiency of $8 \mathrm{bit} / \mathrm{s} / \mathrm{Hz}$ in order to see which transmission mode can achieve the best BER performance. Tab. I shows the quadrature amplitude modulation (QAM) constellations arrangement for each layer. The implementation is based on a measured $(2 \times 2)$ optical MIMO channel using a $1.4 \mathrm{~km}$ multi-mode fiber and transferring at an operating wavelength of $1576 \mathrm{~nm}$ [7]. Here, spatial multiplexing is 
TABLE I. TRANSMISSION MODES

\begin{tabular}{lcc}
\hline throughput & layer 1 & layer 2 \\
\hline $8 \mathrm{bit} / \mathrm{s} / \mathrm{Hz}$ & 256 & 0 \\
$8 \mathrm{bit} / \mathrm{s} / \mathrm{Hz}$ & 64 & 4 \\
$8 \mathrm{bit} / \mathrm{s} / \mathrm{Hz}$ & 16 & 16 \\
\hline
\end{tabular}

realized by transferring multiple modulated light signals over different optical mode groups through a single multi-mode fiber (MMF) [2], [16]. The excitation of different optical mode groups is carried out by varying the light launch eccentricity. However, launching two different light sources into a single MMF with the desired eccentricities is practically complex at the present time. In this work fusion couplers are used for mode combining and splitting [16].

Applying PSVD to this frequency-selective MIMO channel results in layers having a time-dispersive characteristic and hence inter-symbol interference (ISI) occurs on each layer. The ISI is removed by applying a T-spaced zero forcing (ZF) equalizer and therefore this equalization scheme is entitled TPMSVD. The equalizers modify the noise power on each layer differently, which is expressed by the weighting factors $\theta_{\ell}$, with $\ell$ denoting the layer index. These factors determine the layer specific SNRs and hence also the total BER performance [7]. In this example, the noise weighting factors for each layer are computed as $\theta_{1}=37.22$ and $\theta_{2}=4243.46$. In addition, the remaining off-diagonal energy $\varepsilon$, defined as $\varepsilon=\sum_{\tau}\|\mathbf{C}[\tau]\|_{\mathrm{F}}^{2}-\sum_{\tau}\|\boldsymbol{\Sigma}[\tau]\|_{\mathrm{F}}^{2}$, is given by $1.26 \times 10^{-6}$. The value of $\varepsilon$ is negligibly small compared with the input energy, which means that the $\mathrm{CI}$ has been significantly eliminated.

The BER performance results, obtained by applying the MS-SBR2 algorithm for calculating the PSVD, are depicted in Fig. 4. The $(256,0)$ transmission scheme shows the best performance results. In addition, the benefit of using PA which distributes the available transmit power such that the layer specific SNRs are equal is clearly visible. As the SBR2 and MS-SBR2 algorithms are essentially the same class of PEVD algorithms (but different performance of speed), there is no difference between them in terms of the BER performance. However, the PEVD algorithms can offer an improved BER performance over the prior art, i.e., the STVC with SVD based equalization, which has been shown in the previous paper [7].

\section{CONCLUSION}

We have investigated how the proposed MS-SBR2 algorithm can be used in the application of decomposing the channel matrix of a measured $(2 \times 2)$ broadband optical MIMO system. Furthermore, different transmission schemes have been employed to illustrate the BER simulations. In particular, the power allocation scheme has been utilized to further optimize the BER performance. Simulation results have shown that the activation of all transmission layers does not necessarily lead to the best BER performance. On the contrary, the $(256,0)$ QAM with the T-PMSVD equalization scheme seems to achieve the best performance in the studied example.

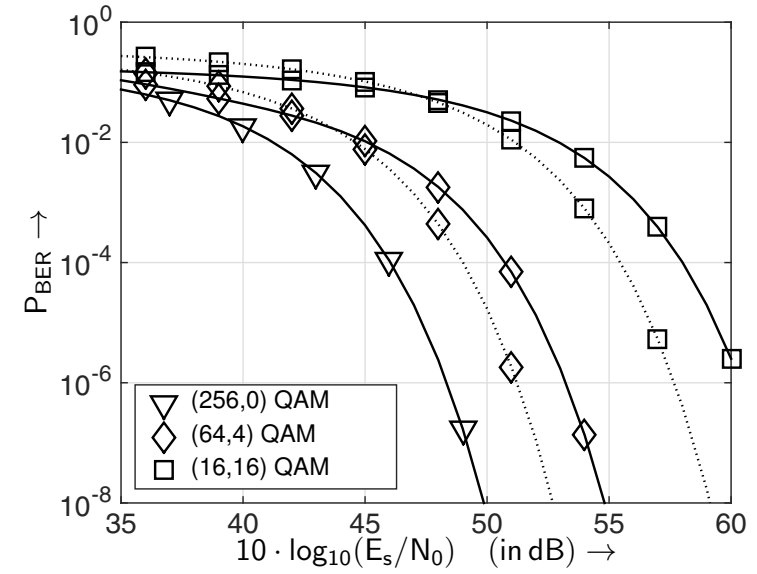

Fig. 4. BER with PA (dotted line) and without PA (solid line) by applying the T-PMSVD equalization scheme, showing the comparisons among different transmission modes when transmitting over the $(2 \times 2)$ optical MIMO channel. Note that no PA is needed for the $(256,0)$ QAM transmission mode.

\section{REFERENCES}

[1] A.C. Singer, N.R. Shanbhag, B. Hyeon-Min, "Electronic Dispersion Compensation - An Overview of Optical Communications Systems," IEEE Signal Processing Magazine, 25(6):110-130, 2008.

[2] P.J. Winzer, G.J. Foschini, "MIMO Capacities and Outage Probabilities in Spatially Multiplexed Optical Transport Systems," Optics Express, 19(17):16680-16696, 2011.

[3] G.C. Raleigh, J.M. Cioffi, "Spatio-temporal Coding for Wireless Communication," IEEE Trans. Communications, 46(3):357-366, Mar 1998.

[4] G.C. Raleigh, V.K. Jones, "Multivariate Modulation and Coding for Wireless Communication," IEEE Journal on Selected Areas in Communications, 17(5):851-866, Mar 1999.

[5] S.S. Haykin, Adaptive Filter Theory, 2nd ed., Upper Saddle River, NJ: Prentice-Hall, 1991.

[6] C.H. Ta, S. Weiss, "A Design of Precoding and Equalisation for Broadband MIMO Systems," in Asilomar Conf. Signals, Systems \& Computers, Pacific Grove, CA, pp. 1616-1620, Nov. 2007.

[7] A. Sandmann, A. Ahrens, S. Lochmann, "Resource Allocation in SVDAssisted Optical MIMO Systems using Polynomial Matrix Factorization," ITG-Fachtagung: Photonische Netze, Leipzig, Germany, 2015.

[8] J.A. Foster, J.G. McWhirter, M.R. Davies, J.A. Chambers, "An Algorithm for Calculating the QR and Singular Value Decompositions of Polynomial Matrices," IEEE Trans. SP, 58(3):1263-1274, Mar. 2010.

[9] J.G. McWhirter, "An Algorithm for Polynomial Matrix SVD Based on Generalised Kogbetliantz Transformations," in 18th EUSIPCO, pp. 457461, Aalborg, Denmark, Aug. 2010.

[10] J.G. McWhirter, P.D. Baxter, "A Novel Technique for Broadband Singular Value Decomposition," in 12th Annиal ASAP Workshop, MA, USA, Mar. 2004

[11] J.G. McWhirter, P.D. Baxter, T. Cooper, S. Redif, J. Foster, "An EVD Algorithm for Para-Hermitian Polynomial Matrices," IEEE Trans. SP, 55(5):2158-2169, May 2007

[12] Z. Wang, J.G. McWhirter, J. Corr, S. Weiss, "Multiple Shift Second Order Sequential Best Rotation Algorithm for Polynomial Matrix EVD," in 23rd EUSIPCO, pp. 849-853, Nice, France, Aug. 2015.

[13] P. P. Vaidyanathan, Multirate Systems and Filter Banks, Prentice-Hall, 1993.

[14] S. Redif, S. Weiss, J.G. McWhirter, "Sequential Matrix Diagonalization Algorithms for Polynomial EVD of Parahermitian Matrices," IEEE Trans. SP, 63(1):81-89, Jan. 2015.

[15] J. Corr, K. Thompson, S. Weiss, J.G. McWhirter, S. Redif, I.K. Proudler, "Multiple Shift Maximum Element Sequential Matrix Diagonalisation for Parahermitian Matrices," in IEEE SSP Workshop, pp. 312-315, Gold Coast, Australia, Jun. 2014.

[16] A. Sandmann, A. Ahrens, S. Lochmann, "Experimental Description of Multimode MIMO Channels utilizing Optical Couplers," ITGFachbericht 248, Photonische Netze, pp. 125-130, 2014. 